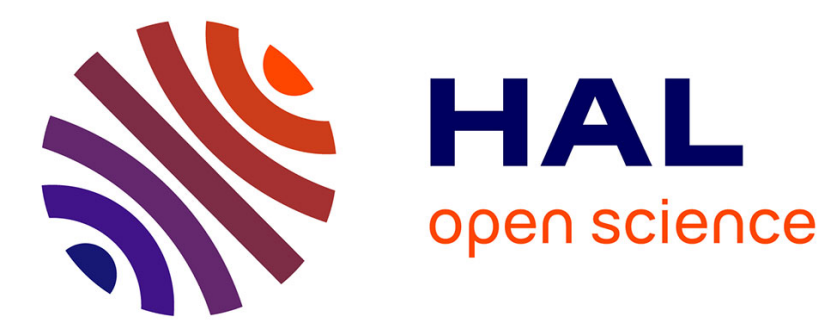

\title{
Entre maître et maîtrise, apprendre le métier Paul Olry
}

\section{To cite this version:}

Paul Olry. Entre maître et maîtrise, apprendre le métier. Enfances \& Psy , 2003. hal-01820277

\section{HAL Id: hal-01820277 \\ https://hal.science/hal-01820277}

Submitted on 3 Mar 2019

HAL is a multi-disciplinary open access archive for the deposit and dissemination of scientific research documents, whether they are published or not. The documents may come from teaching and research institutions in France or abroad, or from public or private research centers.
L'archive ouverte pluridisciplinaire HAL, est destinée au dépôt et à la diffusion de documents scientifiques de niveau recherche, publiés ou non, émanant des établissements d'enseignement et de recherche français ou étrangers, des laboratoires publics ou privés. 


\title{
Entre maître et maîtrise, apprendre le métier
}

\author{
érès | Enfances \& Psy \\ 2003/4 - no24 \\ pages 96 à 102 \\ ISSN 1286-5559
}

Article disponible en ligne à l'adresse:

http://www.cairn.info/revue-enfances-et-psy-2003-4-page-96.htm

Pour citer cet article :

"Entre maître et maîtrise, apprendre le métier", Enfances \& Psy, 2003/4 no24, p. 96-102.

Distribution électronique Cairn.info pour érès.

(c) érès. Tous droits réservés pour tous pays.

La reproduction ou représentation de cet article, notamment par photocopie, n'est autorisée que dans les limites des conditions générales d'utilisation du site ou, le cas échéant, des conditions générales de la licence souscrite par votre établissement. Toute autre reproduction ou représentation, en tout ou partie, sous quelque forme et de quelque manière que ce soit, est interdite sauf accord préalable et écrit de l'éditeur, en dehors des cas prévus par la législation en vigueur en France. II est précisé que son stockage dans une base de données est également interdit. 


\section{Paul Olry \\ Entre maître et maîtrise, apprendre le métier}

Paul Olry est maître

de conférences en Sciences

de l'éducation, université

de Paris 13 et CNAM GRIOT
Apprendre au travail est une évidence pour chacun d'entre nous. Pourtant, travail et éducation s'ignorent encore du point de vue des apprentissages à réaliser. En regard des apprentissages scolaires réputés donner un bagage généraliste, les savoirs du travail sont fréquemment suspectés d'une valeur strictement locale.

Si professionnels et éducateurs s'appliquent à créer les conditions d'un apprentissage des adolescents et jeunes adultes au travail, peu de travaux de pédagogues les y aident. Le champ ainsi laissé libre est occupé par les experts en ressources humaines (Le Boterf, 2000) et les recherches en psychologie du travail et ergonomie (Teiger, 2002 ; Clôt, 2001). Tandis que l'insertion professionnelle des jeunes adultes reste un enjeu politique, peu d'études s'intéressent finalement à l'acquisition des connaissances au travail et au développement des compétences.

Cet article spécifie donc la question centrale de ce numéro « comment apprennent les enfants ? ». Après avoir précisé notre angle d'analyse, nous nous limiterons ici à deux versants du « comment on apprend au travail ». Nous nous intéressons d'abord à l'activité d'agencement des savoirs par les apprentis, recomposition qui conditionne la maîtrise d'un savoir-faire professionnel reconnu. Dans un second temps, nous soulignons l'importance d'une reconnaissance réciproque dans la relation maitre apprenti pour la conquête d'une place par les adolescents.

\section{Apprendre au travail}

Apprendre par l'action est une caractéristique essentielle de l'apprentissage en situation de travail. On sait 
combien cet apprentissage s'inscrit dans une dialectique où le « réussir » précède parfois le « comprendre » (Piaget, 1974). Dans cet esprit, les apprentis sont invités, parfois vertement, par les maîtres d'apprentissage à mettre leurs pas dans les leurs, à prendre enfin en compte la situation quotidienne.

Cette approche «située » de l'action postule que c'est notre démarche qui nous donne un point de vue sur le monde. L'environnement ne fournit pas seulement des contraintes mais aussi des ressources cognitives, à travers les objets et artefacts qu'il comporte : pratiques instituées, formes de pensée, techniques et méthodes.

Cette perspective permet de concevoir l'accomplissement de l'action autrement que dans l'exécution d'instructions, l'application de plans ou l'atteinte d'un résultat comme le fruit d'un calcul, d'une délibération. La pensée s'exprime alors dans l'ordonnancement d'une conduite, dans la production d'un ordre de sens.

En d'autres termes, deux questions retiennent l'attention pour aborder le « comment apprend » l'apprenti : d'une part le rôle respectif des savoirs scolaires et des savoirs de l'action pour la mâ̂trise des situations par le sujet et, d'autre part, le rôle de l'environnement dans la construction d'une identité de professionnel, conscient de ses apprentissages. Ce rapport au savoir bouscule l'identité même de l'individu touché dans ses repères, dans sa pratique. Face aux gestes du métier et aux us et coutumes du travail, les apprentis ont pour seul bagage les savoirs appris à l'école. Apprendre, c'est ainsi sélectionner et confronter avec autrui ce savoir formel, et le transformer, l'ajuster dans une configuration originale et pertinente pour un usage ultérieur.

Cette affirmation est construite sur la base de films commentés ${ }^{1}$ par des apprentis en entreprise, lors de période d'alternance en deuxième année du contrat d'apprentissage. Le cours d'action et son commentaire par l'apprenti permettent de saisir tout ensemble l'organisation de l'activité, ses motifs ${ }^{2}$ et les conditions sociales d'une professionnalisation.

\section{MÉtisser les SAVOIRS POUR MAITTRISER LA SITUATION DE TRAVAIL}

Se demander quels sont les mobiles d'apprentissage d'un adolescent-apprenti qui travaille, c'est s'interroger sur le sens qu'il perçoit entre l'activité de travail et les savoirs dont il dispose. Cette interrogation sur le sens du savoir peut s'énoncer en termes de mobiles : pourquoi apprendre ? En référence à quels modèles et repères identificatoires du métier que l'on veut faire, le savoir
1. Le matériel de l'étude est constitué de douze relevés de cours d'action filmés auprès d'apprentis exerçant dans différents cadres professionnels. S'y ajoutent trois entretiens réalisés en autoconfrontation. Le recueil du matériel s'est effectué à la suite de rencontres de travail dont l'objectif visait pour les participants à produire une analyse de l'action enseignante. Le recueil des données s'est déroulé en 2001.

2. Les éléments présentés sont issus d'une étude pour le ministère de l'Éducation nationale (Astier, Olry, 2001). 
prend-il sens ? C'est le fait de pouvoir donner sens entre ce qui s'apprend à l'école et au travail qui est mobilisateur.

Au travail, l'activité qui « fait sens » résulte en quelque sorte du rapport entre le but visé par le sujet et le motif qui l'incite à agir. Les opérations forment une action efficace (rapport entre les résultats atteints et les buts visés) organisée par la recherche. Du point de vue du « pour quoi apprendre? », notre proposition est que cette organisation de l'activité résulte d'abord d'une capacité réflexive de l'apprenti.

Illustrons notre propos. La préparation du pain traditionnel est une activité courante en boulangerie. La qualité est importante dans la mesure où la baguette « rétro » est vendue plus cher que la baguette blanche. Le but de l'action est donc une préparation soignée de la pâte préformée à l'enfournement. Deux motifs principaux orientent l'action :

- utiliser correctement la force de la pouliche, qui fait lever la pâte et permet au pain de cuire à cour ;

- « faire du beau pain », qui recouvre deux dimensions : la forme générale de la baguette (il ne faut pas qu'elle ressemble à une matraque !) et l'aspect de la croûte, couleur et brillance notamment.

Ces deux motifs lient indissolublement l'efficacité du geste à une appréciation subjective, esthétique de la baguette (simultanément normée et modifiable). La transformation de la pâte en baguette requiert notamment des opérations d'exécution et des opérations d'orientation (Leontiev, 1966).

Pour l'apprenti boulanger, les premières sont inscrites dans la procédure : maintenir la boule de pâte en mouvement au creux de la main lors du façonnage, afin de ne pas altérer une tessiture acquise au repos ; respecter le temps ad hoc de repos après façonnage ; fariner et projeter de la buée dans le four pour la saisie et la brillance de la pâte.

Ces opérations relèvent toutes de la procédure et certaines apparaissent largement maîtrisées et même " routinisées ». Elles trouvent dans des savoirs généraux une justification immédiate.

Les secondes s'inscrivent dans la production d'une expérience validée dans l'action. Elles témoignent d'une forme de maitrise à la jonction des connaissances d'action et des savoirs de l'école.

Ainsi l'alignement et le lamage des baguettes sur le tapis sont des opérations traitant de la maîtrise d'un risque : la déformation. Par delà la procédure, ces opérations, en effet, visent moins à réduire qu'à orienter la déformation des baguettes à la cuisson. La déforma- 
tion agit ainsi comme un organisateur de l'action qui structure l'ordonnancement de l'action. À ce titre, elle devient un concept pragmatique de la conduite de l'action (Pastré, 2002). L'apprenti en exprime des indicateurs. L'action s'énonce alors en termes de connaissances concrètes : « Il faut bien pencher la lame »; exprimées dans un résultat attendu : «Il faut que la baguette crache ! »

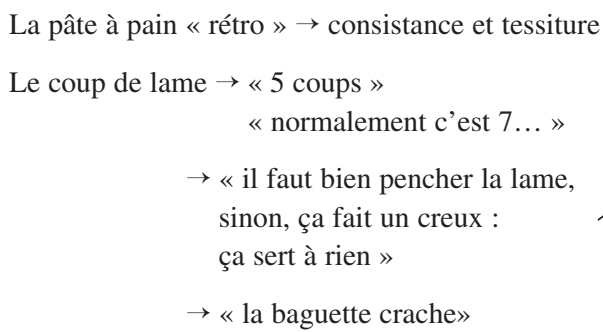

L'alignement des baguettes pour faciliter l'enfournement

\section{Éléments d'une structure de l'activité}

Les opérations de maîtrise ne visent pas seulement à faire du pain, mais du beau pain. Si cette notion est largement véhiculée par les professionnels comme un but, sa portée « organisatrice » est peu perçue. Le sens de l'apprentissage est en revanche construit par l'apprenti autour de la déformation, comme une forme d'agencement, intégrative des différents savoirs. C'est en quelque sorte parce que l'apprenti a traduit un résultat (le beau pain) en un motif (la déformation), que se donne à voir le « comment » il apprend.

Ainsi, tout savoir est une expérience qui se construit. Mais cette construction, acquisition des savoirs d'une pratique ne saurait se faire sans un désir de maîtrise.

\section{MAÎTRISER LA SITUATION POUR AVOIR UNE PLACE AU TRAVAIL}

Si le travail est au cour des processus identitaires, c'est aussi parce qu'il est le lieu où se met en ouvre un projet personnel, où se réalise une trajectoire, où chacun est mis à l'épreuve. On ne peut savoir ce que l'on veut et ce que l'on vaut sans l'avoir expérimenté au travail 3 .

L'effort d'apprentissage est fait non seulement pour maîtriser son activité, pour augmenter son savoir mais aussi pour être reconnu par ses pairs. Dans ce désir de maîtrise, le rôle du maître est essentiel. Il y a toujours désir de maîtrise de celui qui est en situation d'apprentissage, désir de maîtrise qui est désir de reconnaissance médiatisé, c'est-à-dire passant par et exprimé par un faire lorsqu'il s'agit d'un savoir pratique. Geneviève Delbos sou-

La cuisson :

« efficace »

«beau pain »
3. Dubar, C. (1993). «Le travail, lieu et enjeu des constructions identitaires », dans Projet, ${ }^{\circ} 236$, p. 47. 
ligne que la transmission ne peut se faire sans identification à un maître : " Toute personne qui est en situation d'apprentissage est dans une situation où se pose pour elle la question de l'identification à quelqu'un qui est censé être en position de maitrise [...] Si ce processus ne se met pas en marche, je ne vois pas comment il pourrait y avoir transmission car s'en est le moteur même ${ }^{4}$. »

Autrement dit, faire reconnaître ses compétences par le maître, c'est faire montre des qualités éprouvées par l'exercice même du travail. Le développement des compétences est donc tributaire des signes de reconnaissance échangés dans le couple apprenti-maître.

Cette alchimie n'est pas simple comme en témoigne l'exemple d'Aurore, jeune apprentie devant maitriser les situations courantes en sérigraphie. Rappelons rapidement le principe de fabrication : le motif à imprimer est disposé sur un cadre tendu de soie, parcouru d'un système de racles et contreracles qui étalent l'encre en surface pour imprimer un logo, une photo, un slogan, etc. La pression du cadre sur le motif à imprimer et son support conditionnent le résultat esthétique. Selon le maître d'apprentissage, un résultat satisfaisant est le fruit d'une exécution rapide, économique en produit, fiable dans la durée et " beau ».

Le discours d'Aurore met en évidence l'obstacle d'apprentissage que constituent pour elle les diverses conceptions perceptives de la pression : « sentir la pression à la main » (maître d'apprentissage), prendre des repères visuels sur la mise en pression d'un support à encrer (procédural), et la pression atmosphérique enseignée au CFA (déclaratif).

Les images la montrent réglant les cadres métalliques, sans que le plat de sa main ne quitte un instant la trame de soie ainsi que le dicte la norme de métier « sentir la pression à la main ». Pourtant, selon le maître d'apprentissage, Aurore n'est pas en capacité de devenir sérigraphe. Le débat porte entre eux sur la lenteur de l'exécution de sa procédure de calage qu'elle tente de justifier.

A. : On nous a appris... mais vite fait en une minute et...

M.A. : Oui mais c'est pas la peine de s'éterniser dessus puisque quand tu auras pratiqué, que tu auras tiré cent tee-shirts... l'important, c'est ce que tu fais, ce que tu auras senti, puisque tu as un résultat à la fin, qui sera fonction de l'expérience que tu auras acquise...

4. Citée par Roncart, S. (1997). Mémoire de DHEPS, université L.-Pasteur, Strasbourg.
Si la pression est bien au cœur de l'action, c'est la façon d'interpréter la situation qui diverge. Ce qui reste pour Aurore une étape (l'apparition du dessin sur la trame, les contours du motif imprimé, la répartition de l'encre dans le motif), est pour le maître 
d'apprentissage un but, qui peut être atteint par une disposition d'esprit « réflexive », marque du professionnel. Mais le maître d'apprentissage fait bon droit d'un premier niveau de maîtrise : celui du calage des cadres qui va occuper Aurore une heure durant. Aurore sait ce qu'elle ne sait pas, tandis que son maître d'apprentissage sait ce qu'elle devrait savoir.

Donc, ce dernier ne diagnostique rien de cette difficulté dans laquelle Aurore est enfermée. La relation de chacun à l'activité est construite, orientée différemment. Les protagonistes ne peuvent ni trouver les mots pour questionner d'un côté, ni les mots pour expliquer de l'autre. Et cette absence de reconnaissance de soi dans les mots de l'autre rend impossible la transmission des savoirs.

Pourtant, on ne peut gommer ici l'asymétrie de position qui suppose une responsabilité plus grande dans l'étayage de l'acquisition des connaissances par le maître d'apprentissage. Mais, sur les lieux du travail, le processus d'apprentissage est activé par l'enjeu de la maîtrise professionnelle. Les apprentis sont face à des adultes qui maîtrisent un savoir. Ils ne sont plus seulement des adolescents, mais doivent, en professionnels, s'approcher des adultes compétents pour qu'il y ait transmission. La condition pour que s'instaure cette transmission est qu'un espace de reconnaissance soit ouvert à l'apprentie et occupé par elle. Mais occuper cet espace suppose de créer une distance que peut combler son désir de mâ̂trise de ce qui se passe sur le lieu du travail.

Apprendre le métier, c'est en effet acquérir un capital culturel. Le geste technique est aussi un geste social et son appropriation constitue pour l'apprenti, l'établissement d'un rapport de reconnaissances avec ses pairs. Plutôt qu'inculcation, passation des moyens ou des secrets de la maîtrise, la transmission ainsi envisagée est plutôt passation d'une place (ou d'un pouvoir) censée être une place de maîtrise. Ce qui implique que l'apprenti(e) se saisisse et dépasse les actes d'attributions d'identité venant d'autrui, par une revendication d'appartenance et de qualités professionnelles.

\section{ConCLusion}

Nous avons ici souligné deux aspects du « comment on apprend » au travail. D'une part, nous avons vu à quel point apprendre, produire des savoirs, s'inscrit dans une dialectique activité/situation. La production de savoirs s'avère moins portée par l'amont scolaire que résultant d'une investigation progressive. L'activité de l'apprenti est ainsi un moyen de déterminer la situation en «y agissant ». C'est dans la mesure où cette action permet l'engagement dans la situation qu'il est en position de percevoir le « pourquoi apprendre » et de construire un apprentissage. 


\section{Mots-clés}

Activité, situation, apprendre, apprenti, identité.
D'autre part, apprendre au travail suppose l'établissement d'un relation basée sur « un espoir de reconnaissance (salariale et personnelle) qui s'échange contre une attente de mobilisation au travail, incluant plus ou moins d'identification à l'entreprise ou à un groupe professionnel» (Roncart, 1999). Apprendre n'est alors pas une « action sur » mais une « relation entre » un maître et son apprenti. Si la connaissance de l'environnement est utile, c'est moins pour connaître les caractéristiques de la tâche et des processus de maîtrise, que d'offrir un espace de reconnaissance dans lequel chacun ait sa place. Ainsi, la façon dont Aurore gère et utilise ces ressources, s'inspire des modèles d'identification véhiculés tant par le maître d'apprentissage que par le CFA. Apprendre en situation suppose donc que chaque apprenti introduise une distance au modèle pour devenir lui-même, passant de l'identification à l'identisation, capacité « à se défaire d'une histoire d'identifications successives... pour se faire soi » (Tap, 1986).

\section{BIBLIOGRAPHIE}

BORDIGONI, M. 1995. " Jeunes en apprentissage : un besoin de confiance ", CEREQ, BREF, $n^{\circ} 106$.

DelBos, P. ; JoRION, G. 1990. La transmission des savoirs, Paris, Éditions de la MSH.

KUNEGEL, P. 1997. Du maître à l'apprenti : le cas de la réparation automobile, Mémoire DEA CNAM-CRF.

OLRY, P. ; AsTIER, P. 2001. La mobilisation des savoirs généraux par les apprentis en situation de travail, Rapport au ministère de l'Éducation nationale, Paris, CNAM.

PASTRE, P. 1999. Apprendre des situations, Éducation permanente, $n^{\circ} 139$.

PeYRaRd, C. 1990. « La production de connaissances dans l'activité de travail : technologies intellectuelles et pratiques communicationnelles ", L'orientation scolaire et professionnelle, p. 215-230.

RABARDEL, P. 1999. " La composante ergonomique des formations professionnelles et techniques ", Technologies et formation, n 82, p. 4-7.

RONCART, S. 1997. Les apprentis entre apprentissages et identité, Mémoire de DHEPS, Université L.-Pasteur, Strasbourg.

SCHÖN, D. 1996. Le praticien réflexif, Paris, Éditions sociales.

TAP, P. 1986. «L'identification est-elle une aliénation de l'identité ?, dans Identité individuelle et personnalisation, Paris, Privat, Maison des sciences de I'Homme, $2^{\mathrm{e}}$ édition, p. 37-249.

\section{RÉSUMÉ}

Comment les apprentis, adolescents, apprennent-ils dans les situations de travail ? C'est l'agencement des savoirs qui fait ressource dans les situations de travail. Plus que des savoirs, il relève d'abord de l'organisation de l'action au sens strict, puis de l'identité professionnelle et de la place dans l'organisation sociale du travail. La nature des apprentissages réalisés conduit à interroger les conditions de cette transmission, notamment les capacités réflexives des individus apprenants, tant du point de vue du parcours à réaliser que des gestes professionnels à acquérir. 